\title{
Fast and Slow Compaction in Sedimentary Basins
}

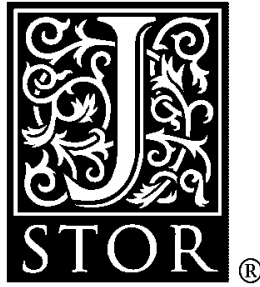

\author{
Andrew C. Fowler; Xin-She Yang
}

SIAM Journal on Applied Mathematics, Vol. 59, No. 1. (Sep. - Oct., 1998), pp. 365-385.

Stable URL:

http://links.jstor.org/sici?sici=0036-1399\%28199809\%2F10\%2959\%3A1\%3C365\%3AFASCIS\%3E2.0.CO\%3B2-M

SIAM Journal on Applied Mathematics is currently published by Society for Industrial and Applied Mathematics.

Your use of the JSTOR archive indicates your acceptance of JSTOR's Terms and Conditions of Use, available at

http://www.jstor.org/about/terms.html. JSTOR's Terms and Conditions of Use provides, in part, that unless you have obtained prior permission, you may not download an entire issue of a journal or multiple copies of articles, and you may use content in the JSTOR archive only for your personal, non-commercial use.

Please contact the publisher regarding any further use of this work. Publisher contact information may be obtained at http://www.jstor.org/journals/siam.html.

Each copy of any part of a JSTOR transmission must contain the same copyright notice that appears on the screen or printed page of such transmission.

The JSTOR Archive is a trusted digital repository providing for long-term preservation and access to leading academic journals and scholarly literature from around the world. The Archive is supported by libraries, scholarly societies, publishers, and foundations. It is an initiative of JSTOR, a not-for-profit organization with a mission to help the scholarly community take advantage of advances in technology. For more information regarding JSTOR, please contact support@jstor.org. 


\title{
FAST AND SLOW COMPACTION IN SEDIMENTARY BASINS*
}

\author{
ANDREW C. FOWLER ${ }^{\dagger}$ AND XIN-SHE YANG ${ }^{\dagger}$
}

\begin{abstract}
A mathematical model of compaction in sedimentary basins is presented and analyzed. Compaction occurs when accumulating sediments compact under their own weight, expelling pore water in the process. If sedimentation is rapid or the permeability is low, then high pore pressures can result, a phenomenon which is of importance in oil drilling operations. Here we show that one-dimensional compaction can be described in its simplest form by a nonlinear diffusion equation, controlled principally by a dimensionless parameter $\lambda$, which is the ratio of the hydraulic conductivity to the sedimentation rate. Large $\lambda$ corresponds to very permeable sediments, or slow sedimentation, a situation which we term "fast compaction," since the rapid pore water expulsion allows the pore water pressure to equilibrate to a hydrostatic value. On the other hand, small $\lambda$ corresponds to "slow compaction," and the pore pressure is in excess above the hydrostatic value and more nearly equal to the overburden value. We provide analytic and numerical results for both large and small $\lambda$, using also the assumption that the permeability is a strong function of porosity. In particular, we can derive Athy's law (that porosity decreases exponentially with depth) when $\lambda \gg 1$.
\end{abstract}

Key words. compaction, sedimentary basins, abnormal pore pressure

AMS subject classifications. 35C20, 35R35, 76S05, 86A60, 86A99

PII. S0036139996287370

1. Introduction. Sedimentary basins, such as those in the North Sea or the Gulf of Mexico, form when waterborne sediments in shallow seas are deposited over periods of tens of millions of years. The resulting sediments, which may, for example, be sands or river muds washed down from land, then compact under their own weight, causing a reduction of porosity (and hence the expulsion of pore water) and eventually (as depth and thus pressure and temperature increase) cementation reactions occur, causing a transformation from a granular aggregate to rocks, such as shales or sandstones.

Sedimentary basins are prime locations for the formation of hydrocarbons and are thus important in the oil industry. One particular problem which affects drilling operations is the occasional occurrence of abnormally high pore fluid pressures, which, if encountered suddenly, can cause drill hole collapse and consequent failure of the drilling operation. An understanding of how such high pore pressures occur is therefore of some industrial, as well as scientific, interest (Bredehoeft and Hanshaw (1968), Bishop (1979)). Furthermore, the variation of porosity with depth is a source of information for geologists who are concerned with understanding the burial and subsidence histories of sedimentary basins (Smith (1971), Lerche (1990)). Compaction models which describe these processes are thus of practical interest.

The basic model of compaction is rather analogous to the process of soil consolidation. The sediments act as a compressible porous matrix, so mass conservation of pore fluid together with Darcy's law leads to an equation of the general type $\phi_{t}+\nabla \cdot \mathbf{q}=0$, $\mathbf{q} \propto-\nabla p$, where $\phi$ is porosity, $\mathbf{q}$ is fluid flux, and $p$ is fluid pressure. This model must be supplemented by a constitutive law relating pore pressure $p$ to porosity $\phi$, and in soil mechanics this takes (most simply) the form of the normal consolidation

\footnotetext{
${ }^{*}$ Received by the editors August 22, 1996; accepted for publication (in revised form) April 25, 1997; published electronically October 29, 1998.

http://www.siam.org/journals/siap/59-1/28737.html

${ }^{\dagger}$ Centre for Industrial and Applied Mathematics, Mathematical Institute, Oxford University, 2429 St. Giles', Oxford OX13LB, UK (fowler@maths.ox.ac.uk).
} 
line, which relates $\phi$ to the effective pressure $p_{e}$ defined by

$$
p_{e}=P-p,
$$

where $P$ is the overburden pressure. Terzaghi's principle of effective stress (Terzaghi (1943)) states that for soils, it is the effective pressure (more generally the effective stress) which controls the deformation, and the extension of this principle to compacting sediments is termed Athy's law, after work by Athy (1930), who measured porosity-depth profiles and proposed that $\phi=\phi\left(p_{e}\right)$. In many cases, an exponential decrease of porosity with depth occurred, and we will sometimes equivocally refer to this kind of profile as an Athy profile.

It can be seen that with this constitutive assumption, the basic compaction model will essentially comprise a nonlinear diffusion equation, and our purpose here is to provide analytic and numerical solutions which provide signposts to the kind of behavior that can be expected in more realistic models.

Two principal kinds of realism are of concern, although we do not analyze them in this paper. The first is diagenesis, which is effectively a dewatering reaction which occurs when smectite (a water-rich clay mineral) dissolves to form quartz in solution together with free water, the quartz subsequently precipitating as illite (a water-free clay). Effectively, the smectite-illite reaction acts as a source for pore water and can thus enhance (or even be a primary cause of) abnormal pore pressures, though to what extent is unknown.

The other complication concerns the assumed rheology. Even for soils, the relationship $\phi=\phi\left(p_{e}\right)$ is irreversible, exhibiting hysteresis, and incorporation of this into basin loading/unloading histories complicates the model conceptually. Furthermore, as pressure increases, pressure solution occurs, as precipitation and dissolution depend on the local grain-to-grain pressure. This leads to an effective creep of the solid grains. Also, calcite precipitation at grain junctions causes cementation and thus stiffening of the solid matrix. These effects, and those of diagenesis, are not considered in this paper.

Early studies of compaction by Athy (1930) and Hedberg (1936) have more recently been followed in work by Gibson (1958); see also Gibson, England, and Hussey (1967) and Gibson, Schiffman, and Cargill (1981). Other recent models of compaction include those of Smith (1971), Keith and Rimstidt (1985), Wangen (1992), Shi and Wang (1986), and Luo and Vasseur (1992). Some of this (and other) work was reviewed by Audet and Fowler (1992), whose formalism we follow here. All of the above papers, however, ignore the complications of diagenesis and realistic rheology. Ricke and Chilingarian (1974) offer a comprehensive review of sedimentary compaction.

Audet and Fowler (1992) provide a general discussion of how diagenesis and, to some extent, exotic rheology, can be included in compaction models. Because the structure of such models is very complicated, their approach was to develop analytic insight into simpler models first, and they were able to find reasonable approximations for large $\lambda$ and large time, and also for small $\lambda$, where $\lambda$ is a dimensionless number which measures the ratio of the hydraulic conductivity of the sediments to the sedimentation rate. Here we extend and improve those results for smaller times, which are of more geological significance.

The term compaction has been much used in the geophysical literature, mainly to describe the extraction of magma from source regions in the earth's mantle. This work has been reviewed by Fowler (1990) and was based on original papers by Scott and Stevenson (1984), McKenzie (1984), and Fowler (1985). The principal difference 


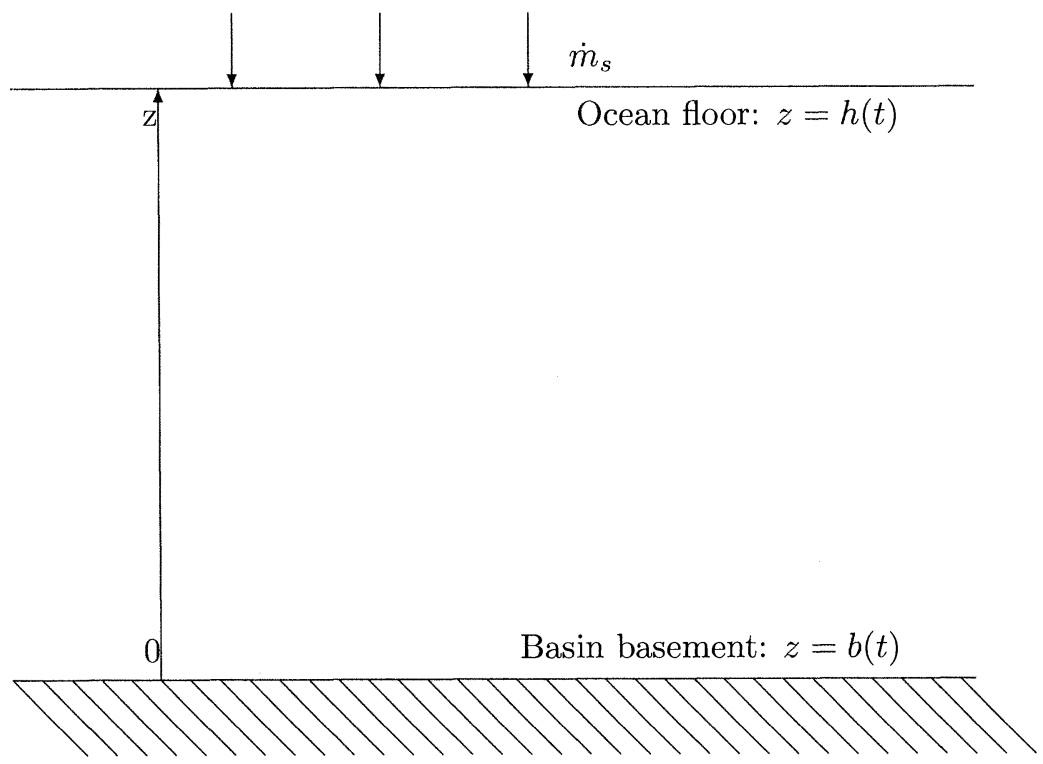

FIG. 1. One-dimensional compacting sedimentary basin. The coordinate $z$ is directed upwards.

between those models and the present one is in the rheology of the solid matrix. The solid grains in the mantle at depths of 100-200 km respond to a differential pressure between overburden and pore pressures by grain creep. This leads to a very different model from the one proposed here, which essentially considers the matrix to deform elastically. Pressure solution creep might be more analogous to the viscous compaction of the mantle, and this has been analyzed by Angevine and Turcotte (1983).

2. Model equations. We consider the solid matrix to behave as an elastic solid, and specifically so that the porosity $\phi$ is a function of effective pressure $p_{e}$. The model describes the one-dimensional flow of both solid and liquid phases and is based on the framework developed by Audet and Fowler (1992). For a one-dimensional basin $b(t)<z<h(t)$, where $h(t)$ is the ocean floor and $b(t)$ is the basement rock as shown in Fig. 1, the governing model equations for one-dimensional compaction can be written as follows.

Mass conservation:

$$
\begin{gathered}
\frac{\partial \phi}{\partial t}+\frac{\partial}{\partial z}\left(\phi u^{l}\right)=0 \\
-\frac{\partial \phi}{\partial t}+\frac{\partial}{\partial z}\left[(1-\phi) u^{s}\right]=0 .
\end{gathered}
$$

Darcy's law:

$$
\phi\left(u^{l}-u^{s}\right)=-\frac{k}{\mu}\left(\frac{\partial p}{\partial z}+\rho_{l} g\right) .
$$

Force balance:

$$
\frac{\partial \sigma_{3}}{\partial z}-\left[\rho_{s}(1-\phi)+\rho_{l} \phi\right] g=0
$$




\section{Constitutive relation:}

$$
p_{e}=p_{e}(\phi)
$$

In these equations, $u^{l}$ and $u^{s}$ are the velocities of fluid and solid matrix, $k$ and $\mu$ are the matrix permeability and the liquid viscosity, $\sigma_{3}$ is the vertical component of the stress tensor, and $g$ is the gravitational acceleration.

We can relate $\sigma_{3}$ to the effective pressure and pore pressure as follows. First of all, we may modify Terzaghi's relation (1) by writing

$$
p_{e}=P-(1-a) p,
$$

a relationship due to Skempton (1960), who suggested that although for soils $a$ might be small due to a low grain-to-grain interfacial contact area, this would not necessarily be the case for a more compacted rocklike matrix. Further discussion of the effective pressure is given by Bear and Bachmat (1990).

In conditions of uniaxial strain, where the only nonzero strain rate is $\partial U / \partial z$, where $U$ is vertical strain, the nonzero components of the effective stress tensor

$$
\boldsymbol{\sigma}^{\prime}=\boldsymbol{\sigma}+(1-a) p \boldsymbol{\delta}
$$

are the diagonal components given for an elastic medium by

$$
\begin{gathered}
\sigma_{1}^{\prime}=\sigma_{e}^{\prime}-\frac{2}{3} G \frac{\partial U}{\partial z} \\
\sigma_{3}^{\prime}=-p_{e}+\frac{4}{3} G \frac{\partial U}{\partial z}
\end{gathered}
$$

where $G$ is the shear modulus.

Now, from (3), we have

$$
\frac{d \phi}{d t_{s}}=(1-\phi) \frac{\partial u^{s}}{\partial z}
$$

where

$$
u^{s}=\frac{\partial U}{\partial t}+u^{s} \frac{\partial U}{\partial z}
$$

and $d / d t_{s}$ is a material time derivative following the solid matrix; thus

$$
\frac{\partial u^{s}}{\partial z}=\frac{\partial \Delta}{\partial t}+\Delta \frac{\partial u^{s}}{\partial z}+u^{s} \frac{\partial \Delta}{\partial z}
$$

where

$$
\Delta=\frac{\partial U}{\partial z}
$$

is the dilation. Thus

$$
\frac{\partial u^{s}}{\partial z}=\frac{1}{1-\Delta} \frac{d \Delta}{d t_{s}},
$$

so that, using (10),

$$
\frac{1-\phi}{1-\Delta}=\text { constant }
$$


following the solid matrix; that is, it is constant in time for each solid matrix element. Since each solid element originates at the surface, where conditions are assumed to be uniform, we can also assume that this expression is constant also in space and equal to $A$, say; then if $G$ is constant and

$$
d p_{e} / d \phi=-K
$$

(which may depend on $\phi$ ), we find

$$
\frac{\partial \sigma_{e}^{\prime}}{\partial z}=-\left(1+\frac{4 G}{3 K A}\right) \frac{\partial p_{e}}{\partial z}
$$

and (5) becomes, using (8),

$$
-\left(1+\frac{4 G}{3 K A}\right) \frac{\partial p_{e}}{\partial z}-(1-a) \frac{\partial p}{\partial z}-\left[\rho_{s}(1-\phi)+\rho_{l} \phi\right] g=0 .
$$

2.1. Boundary conditions. These are five equations for five unknown variables: one for porosity $\phi$, two for velocities $u^{s}, u^{l}$, and two for effective pressure $p_{e}$ and pore pressure $p$. The system is of fourth order, so we will require boundary conditions on $u^{l}, u^{s}, p, p_{e}$; in addition, we assume $b(t)$ is known but $h(t)$ is not, which is therefore described by a further boundary condition. The natural boundary conditions are the kinematic boundary conditions at $z=b$,

$$
u^{s}=u^{l}=\dot{b},
$$

and a kinematic condition at $z=h$,

$$
\dot{h}=\dot{m}_{s}+u^{s}
$$

where $\dot{m}_{s}$ is the sedimentation rate at $z=h$. Also at $z=h$,

$$
\phi=\phi_{0}, p=p_{0}
$$

where $p_{0}$ is the overburden pressure, e.g., due to ocean depth. $\phi_{0}$ is the value at the top of basin during sedimentation. Equation (20) gives $h$, and then we have four conditions for $u^{l}, u^{s}, p, p_{e}$ as required.

The choice of $\phi_{0}$ will normally follow from the constitutive relation $p_{e}=p_{e}(\phi)$, for example, if we take $p_{e}=0$ at $z=h$. The value of $A$ then follows from a normal stress balance, since we require also $-\sigma_{3}^{\prime}=p_{0}$, which implies

$$
\frac{4}{3} G \Delta=p_{e}\left(\phi_{0}\right)-a p_{0}
$$

at $z=h$. For example, the reasonable assumption $p_{e}=0, a=0$ implies $\Delta=0$ and thus $A=1-\phi_{0}$ (everywhere).

2.2. Nondimensionalization. A natural depth scale to choose is that over which $\phi$ changes significantly. Since $p_{e}=p_{e}(\phi)$, we can equivalently define a pressure scale over which $\phi$ changes significantly. To be specific, define a pressure scale $[p]$ by writing

$$
p_{e}(\phi)=[p] \tilde{p}(\phi),
$$


where $[p]$ is such that $\tilde{p}$ varies by $O(1)$ when $\phi$ does. Since the variation of $p_{e}$ is determined by (18), we can equivalently choose a depth scale $d$ by putting

$$
\left(1+\frac{4 G}{3 K A}\right)[p]=\left(\rho_{s}-\rho_{l}\right) g d .
$$

Here we assume that $G / K$ is constant, which may be a reasonable assumption. Let $\dot{m}_{s}^{0}$ be a typical value of the (positive) sedimentation rate. We now scale the variables by writing

$$
\begin{aligned}
z & =d z^{*}, \quad h=d h^{*}, \quad b=d b^{*}, \\
u^{s} & =\dot{m}_{s}^{0} u^{s *}, \\
u^{l} & =\dot{m}_{s}^{0} u^{l *}, \\
t & =\left(d / \dot{m}_{s}^{0}\right) t^{*}, \\
\dot{m}_{s} & =\dot{m}_{s}^{0} \dot{m}_{s}^{*}, \\
p & =p_{0}+\left(\rho_{s}-\rho_{l}\right) g d p^{*}, \\
k & =k_{0} \tilde{k}(\phi), \quad k_{0}=k\left(\phi_{0}\right) .
\end{aligned}
$$

These variables are substituted into the equations, which then become, on dropping the asterisks for further convenience,

$$
\begin{gathered}
\frac{\partial \phi}{\partial t}+\frac{\partial}{\partial z}\left(\phi u^{l}\right)=0 \\
-\frac{\partial \phi}{\partial t}+\frac{\partial}{\partial z}\left[(1-\phi) u^{s}\right]=0 \\
\phi\left(u^{l}-u^{s}\right)=-\lambda \tilde{k}\left(\frac{\partial p}{\partial z}+r\right), \\
-\frac{\partial \tilde{p}}{\partial z}-(1-a) \frac{\partial p}{\partial z}-(1+r)+\phi=0 \\
\tilde{p}=\tilde{p}(\phi)
\end{gathered}
$$

where

$$
\lambda=\frac{k_{0}\left(\rho_{s}-\rho_{l}\right) g}{\mu \dot{m}_{s}^{0}}, r=\frac{\rho_{l}}{\rho_{s}-\rho_{l}} .
$$

The boundary conditions take the same form as in (19)-(21), except that $p=0$ at $z=h$. We add the first two equations of mass conservation together and integrate from 0 to $z$; thus

$$
\phi u^{l}+(1-\phi) u^{s}=\dot{b} .
$$

By using Darcy's law, we obtain

$$
u^{s}=\lambda \tilde{k}\left(\frac{\partial p}{\partial z}+r\right)+\dot{b} .
$$


The boundary conditions can thus be written in the form

$$
\begin{gathered}
\frac{\partial p}{\partial z}+r=0 \text { at } z=b, \\
\phi=\phi_{0}, \quad \dot{h}=\dot{m}_{s}+\lambda \tilde{k}\left(\frac{\partial p}{\partial z}+r\right)+\dot{b} \text { at } z=h .
\end{gathered}
$$

2.3. Excess pore pressure. The hydrostatic pressure at $z$ is defined as

$$
p_{h}=p_{0}+\int_{z}^{h(t)} \rho_{l} g d z .
$$

The overburden pressure (strictly, the normal stress) at $z$ is defined as

$$
P=p_{0}+\int_{z}^{h(t)}\left[(1-\phi) \rho_{s}+\phi \rho_{l}\right] g d z .
$$

The excess pore pressure or abnormal overpressure $p_{\text {ex }}$ is defined as

$$
p_{\text {ex }}=p-p_{h},
$$

which is the pressure in excess of the hydrostatic pressure.

By using these definitions and employing the force balance equation (29), the dimensionless differential forms of the above definitions are

$$
\begin{gathered}
-\frac{\partial P}{\partial z}=1+r-\phi, \\
-\frac{\partial p_{h}}{\partial z}=r, \\
-(1-a) \frac{\partial p_{\mathrm{ex}}}{\partial z}=\frac{\partial \tilde{p}}{\partial z}+(1+a r-\phi) .
\end{gathered}
$$

2.4. A general nonlinear diffusion equation. By using (29), (32), and (33), (27) reduces to a nonlinear diffusion equation for $\phi$ :

$$
\frac{\partial \phi}{\partial t}=\frac{\lambda}{1-a} \frac{\partial}{\partial z}\left\{\tilde{k}(1-\phi)\left[-\tilde{p}^{\prime}(\phi) \frac{\partial \phi}{\partial z}-(1+a r-\phi)\right]\right\}-\dot{b} \frac{\partial \phi}{\partial z} .
$$

The boundary conditions are then

$$
\begin{gathered}
-\tilde{p}^{\prime}(\phi) \frac{\partial \phi}{\partial z}-(1+a r-\phi)=0 \text { at } z=b, \\
\phi=\phi_{0} \text { at } z=h, \\
\dot{h}=\dot{m}_{s}-\frac{\lambda}{1-a} \tilde{k}\left[\tilde{p}^{\prime}(\phi) \frac{\partial \phi}{\partial z}+(1+a r-\phi)\right]+\dot{b} \text { at } z=h .
\end{gathered}
$$

Since in practice $\tilde{p}^{\prime}(\phi)<0$, we see that (42) is a nonlinear diffusion equation, valid in the domain $b<z<h$, where $h$ is unknown and is determined by the extra boundary condition in (43). The problem is thus one of free boundary type. 
2.5. Determination of model parameters. Of the parameters appearing in (42) and (43), $r$ and $a$ are $O(1)$ constants which are essentially fixed as material properties. The important parameter which controls compaction is the compaction number $\lambda$. We estimate its size using observations given by other authors (Smith (1971), Sharp (1976), Sharp and Domenico (1976), Eberl and Hower (1976), Bethke and Corbet (1988), Lerche (1990), Audet and Fowler (1992)). For example, if we take $d \sim 1 \mathrm{~km}, k_{0} \sim 1 \times 10^{-18} \mathrm{~m}^{2}, \rho_{s} \sim 2.6 \times 10^{3} \mathrm{~kg} \mathrm{~m}^{-3}, g \sim 10 \mathrm{~m} \mathrm{~s}^{-2}, \rho_{l} \sim 1 \times 10^{3} \mathrm{~kg}$ $\mathrm{m}^{-3}, \mu \sim 1 \times 10^{-3} \mathrm{~N} \mathrm{~s} \mathrm{~m}^{-2}, \dot{m}_{s}^{0} \sim 300 \mathrm{~m} \mathrm{Ma}^{-1}=1 \times 10^{-11} \mathrm{~m} \mathrm{~s}^{-1}$; then $\lambda \approx 1$ and $r \approx 0.63$. Typical values of the uncompacted permeability $k_{0}$ are given by Freeze and Cherry (1979). The permeability is proportional to the square of the grain size, with a typical proportionality factor of $10^{-4}$ which allows for tortuosity and constriction of the pore space. Marine clays (particle size less than $2 \mu \mathrm{m}$ ) have permeabilities in the range $10^{-16}-10^{-19} \mathrm{~m}^{2}$, silts (particle size 2-60 $\mu \mathrm{m}$ ) have permeabilities $10^{-12}-10^{-16}$ $\mathrm{m}^{2}$, while sands $(60 \mu \mathrm{m}-2 \mathrm{~mm})$ have permeabilities $10^{-9}-10^{-13} \mathrm{~m}^{2}$. Cemented clay forms shale, cemented sand forms sandstone, and these have somewhat lower permeabilities than the corresponding uncemented matrix. We see that a wide range of permeabilities between $10^{-9} \mathrm{~m}^{2}$ and $10^{-19} \mathrm{~m}^{2}$ can occur, so values of $\lambda$ may lie in the range $10^{-1}-10^{9}$. Values of $\lambda$ which are either small or large are therefore of interest, although that of large $\lambda$ is the more likely. This is also the more interesting case mathematically. An initial porosity of $\phi_{0}=0.5$ at the top of the basin is used by other authors (Smith (1971), Sharp (1976), Bethke and Corbet (1988), Audet and Fowler (1992)).

2.6. Simplification of the nonlinear diffusion equation. There is no loss of generality in choosing $\dot{b}=0$ so that $z=0$ denotes the basement. Skempton (1960) suggested that $a$ is small, and in what follows we take $a=0$ without expecting that this choice will have a major effect on the solutions. Based on the work of Smith (1971), Sharp (1976), and Audet and Fowler (1992), we adopt the following constitutive functions:

$$
\begin{gathered}
\tilde{p}=\ln \left(\phi_{0} / \phi\right)-\left(\phi_{0}-\phi\right), \\
\tilde{k}=\left(\phi / \phi_{0}\right)^{m}, \quad m=8, \\
\dot{m}_{s}=1 .
\end{gathered}
$$

With these simplifications, the nonlinear diffusion equation for $\phi$ can be written in a compact form as

$$
\frac{\partial \phi}{\partial t}=\lambda \frac{\partial}{\partial z}\left\{\tilde{k}(1-\phi)^{2}\left[\frac{1}{\phi} \frac{\partial \phi}{\partial z}-1\right]\right\}
$$

with boundary conditions

$$
\begin{gathered}
\phi_{z}-\phi=0 \text { at } z=0 \\
\phi=\phi_{0}, \quad \dot{h}=1+\lambda \tilde{k}(1-\phi)\left[\frac{1}{\phi} \frac{\partial \phi}{\partial z}-1\right] \text { at } z=h .
\end{gathered}
$$

The analysis of this model forms the subject of the rest of this paper. In addition, the problem is also solved numerically on a normalized grid $Z=z / h(t)$, by using the predictor-corrector implicit finite-difference method presented by Meek and Norbury (1982), to make a comparison with the obtained analytic solutions. 
3. Analysis. We expect that values of $\lambda$ will usually lie in the range $10^{-2}-10^{3}$. Since $\lambda$ is the controlling parameter which characterizes the compaction behavior, we can expect that $\lambda=1$ defines a transition between slow sedimentation (fast compaction) $\lambda>>1$ and fast sedimentation (slow compaction) $\lambda<<1$ and that the evolution features of fast and slow compaction also may be quite different.

3.1. Slow compaction $(\boldsymbol{\lambda}<<1$ ). For $\lambda \ll 1, z \sim 1$, and $t \sim 1$, (47) implies that $\partial \phi / \partial t \approx 0$, so with $\phi=\phi_{0}$ on $z=h$, then $\phi \approx \phi_{0}$ and $\tilde{k} \approx 1$ for $z>0$. Furthermore, we see that $h \approx t$. The boundary condition at the base is not satisfied, and a boundary layer is necessary there. For sufficiently small times, we can take $\phi \approx \phi_{0}$ near $z=0$ as well so that (47) may be approximated (uniformly) as

$$
\frac{\partial \phi}{\partial t}=\lambda^{\prime} \frac{\partial^{2} \phi}{\partial z^{2}}, \quad \lambda^{\prime}=\lambda \frac{\left(1-\phi_{0}\right)^{2}}{\phi_{0}},
$$

with appropriate boundary conditions for the basal boundary layer being

$$
\frac{\partial \phi}{\partial z}-\phi=0 \text { on } z=0
$$

$$
\phi \rightarrow \phi_{0} \text { as } z \rightarrow \infty
$$

where the latter represents the matching condition outside the boundary layer in which $z \sim \lambda^{1 / 2}$. The solution can be easily obtained by a standard Laplace transformation method (Carslaw and Jaeger (1959)) as

$$
\phi=\phi_{0} \operatorname{erf}\left[\frac{z}{\left(4 \lambda^{\prime} t\right)^{1 / 2}}\right]+\phi_{0} e^{z+\lambda^{\prime} t} \operatorname{erfc}\left[\frac{z}{\left(4 \lambda^{\prime} t\right)^{1 / 2}}+\left(\lambda^{\prime} t\right)^{1 / 2}\right] .
$$

We see that the assumption that $\phi$ is close to $\phi_{0}$ is self-consistent for $t \ll 1 / \lambda^{\prime}$ and in particular for times (of interest) of $O(1)$. In fact, expansion of (53) with $\eta=z / 2\left(\lambda^{\prime} t\right)^{1 / 2}=O(1)$ and $\lambda^{\prime} t$ small shows that $\phi=\phi_{0}+O\left[\left(\lambda^{\prime} t\right)^{1 / 2}\right]$. The solution indicates that compaction develops only in a small range near the basin basement, with a thickness growing with $\sqrt{\lambda^{\prime} t}$. When $a=0$, we are in the case discussed by Audet and Fowler (1992) with a similarity solution (their equation (5.26)).

Audet and Fowler (1992) gave a slightly different result for this case by putting $\phi-z=\phi_{0}$ on $z=0$. While their result is asymptotically equivalent to (53) for $\lambda^{\prime} t \ll 1$, it is likely to be less accurate for larger times, a fact which is confirmed by numerical integration, as shown in Fig. 2.

The dimensionless overburden, hydrostatic, and excess pore pressures satisfy, respectively,

$$
-\frac{\partial P}{\partial z}=1+r-\phi
$$

$$
-\frac{\partial p_{h}}{\partial z}=r
$$

$$
-\frac{\partial p_{\mathrm{ex}}}{\partial z}=(1-\phi)\left(1-\phi_{z} / \phi\right) .
$$




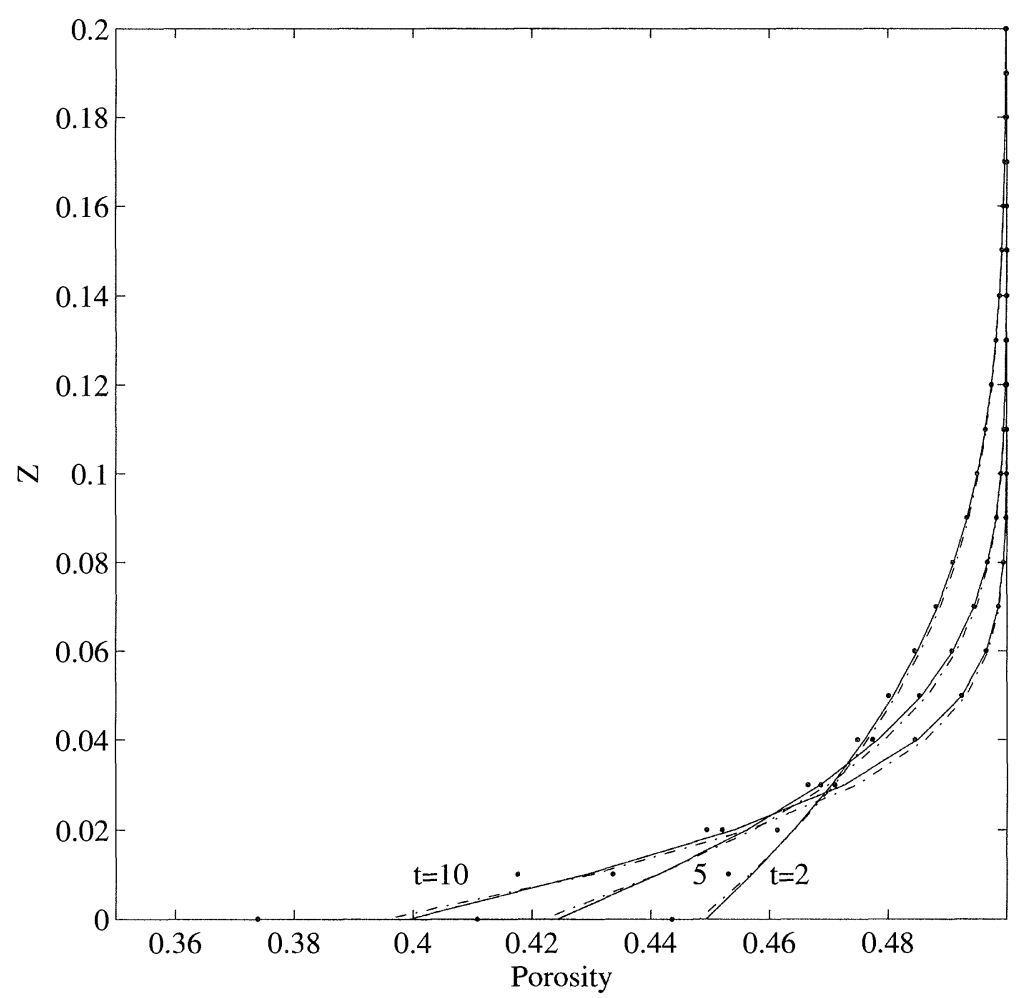

FIG. 2. Comparison of solutions of (47)-(49) in the vicinity of the basement $z=0$ in terms of the normalized height $Z=z / h(t)$, for $\lambda=0.01$. The solid lines represent a direct numerical solution, while the dashed lines are the solutions of (50)-(53). Audet and Fowler's further approximation (1992, eq.(5.26)) is shown as the dotted profiles. It can be seen that the dotted profile deviates from the correct solution at larger values of $t$.

It follows that

$$
p_{\mathrm{ex}}=\int_{z}^{h}(1-\phi) d z-\ln \left(\phi_{0} / \phi\right)+\phi_{0}-\phi
$$

and hence, for $\lambda^{\prime} t \ll 1$, we have the leading-order solution $p_{\mathrm{ex}} \approx\left(1-\phi_{0}\right)(h-z)$. The other terms are only small corrections. The excess pressure develops proportionally to basin thickness.

A comparison of the above solution with the numerical results is plotted in Fig. 3. It can be seen that the agreement is very good and that, for $\lambda<<1$, overpressure is essentially proportional to basin thickness.

3.2. Fast compaction $(\lambda>>1)$. For large values of $\lambda$, we assume expansions of the form

$$
\begin{aligned}
& \phi=\phi^{(0)}+\frac{1}{\lambda} \phi^{(1)}+\frac{1}{\lambda^{2}} \phi^{(2)}+\cdots . \\
& h=h^{(0)}+\frac{1}{\lambda} h^{(1)}+\frac{1}{\lambda^{2}} h^{(2)}+\cdots .
\end{aligned}
$$




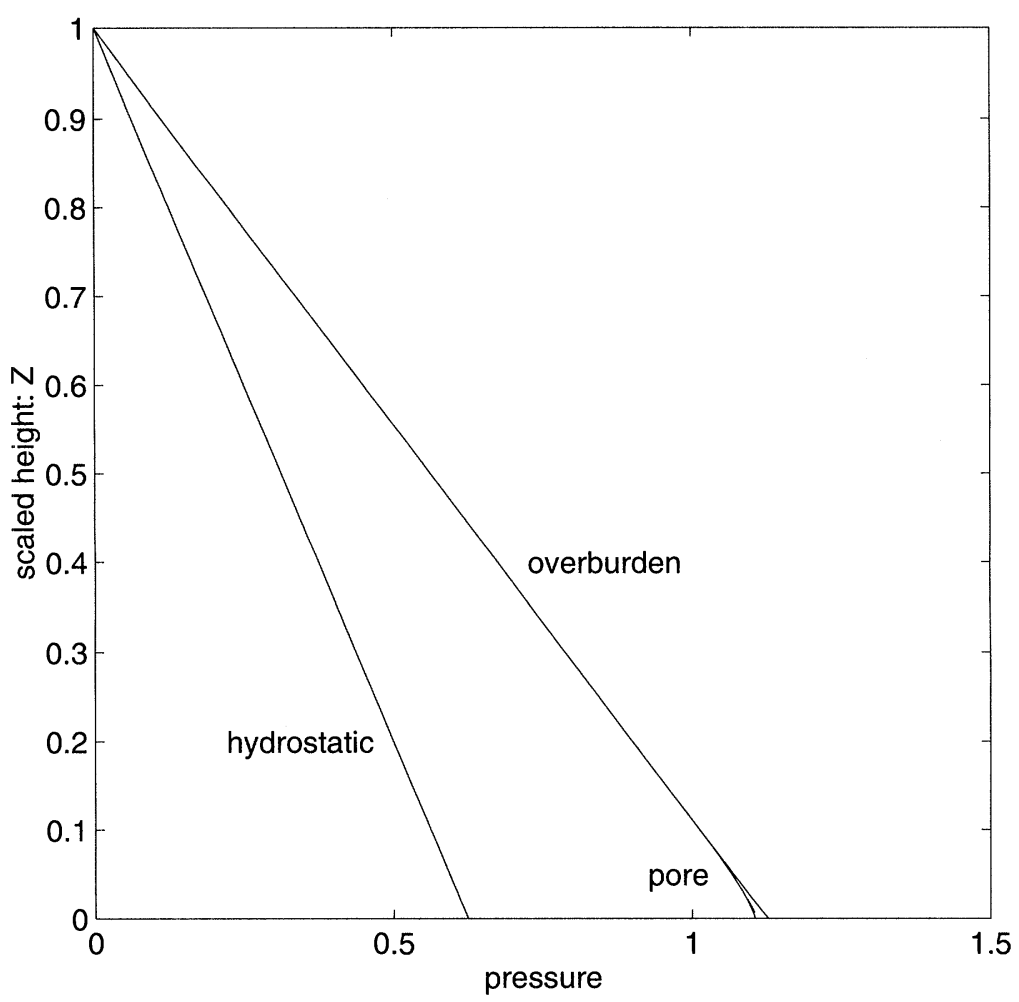

FIG. 3. Hydrostatic, pore, and overburden pressures at $t=5$, using values $r=0.63$ and $\lambda=0.01$. Solid lines correspond to numerical results; the dashed line for $p_{\mathrm{ex}}$ is calculated from the solution (57). The numerical and analytical results are indistinguishable.

Substituting the above expansions into (47)-(49) and equating the coefficients of powers of $1 / \lambda$, we have

$$
\begin{gathered}
\frac{\partial}{\partial z}\left\{\tilde{k}^{0}\left(1-\phi^{(0)}\right)^{2}\left[\frac{1}{\phi^{(0)}} \phi_{z}^{(0)}-1\right]\right\}=0, \\
\phi_{t}^{(0)}=\frac{\partial}{\partial z}\left\{\tilde{k}^{0}\left(1-\phi^{(0)}\right)^{2} \frac{1}{\phi^{(0)}}\left[\phi_{z}^{(1)}-\frac{\phi^{(1)}}{\phi^{(0)}} \phi_{z}^{(0)}\right]\right\},
\end{gathered}
$$

where $\tilde{k}^{0}=\left(\phi^{(0)} / \phi_{0}\right)^{m}$ and we have in (61) anticipated the result in (65) below. The boundary conditions become the following.

At $z=h^{(0)}$ :

$$
\begin{gathered}
\phi^{(0)}=\phi_{0}, \\
\phi^{(1)}+h^{(1)} \phi_{z}^{(0)}=0 .
\end{gathered}
$$

At $z=0$ :

$$
\phi_{z}^{(0)}=\phi^{(0)}
$$




$$
\phi_{z}^{(1)}=\phi^{(1)}
$$

with

$$
\dot{h}^{(0)}=1+\tilde{k}^{0}\left(1-\phi^{(0)}\right) \frac{1}{\phi^{(0)}}\left[\phi_{z}^{(1)}-\frac{\phi^{(1)}}{\phi^{(0)}} \phi_{z}^{(0)}\right]
$$

on $z=h^{(0)}$, where again we use (60) to anticipate (65).

Integrating equation (60) and using the boundary condition (63), we have

$$
\tilde{k}^{0}\left(1-\phi^{(0)}\right)^{2}\left[\frac{1}{\phi^{(0)}} \phi_{z}^{(0)}-1\right]=0
$$

and hence

$$
\frac{1}{\phi^{(0)}} \phi_{z}^{(0)}-1=0 \text { with } \phi^{(0)}=\phi_{0} \text { at } z=h^{(0)} .
$$

Its solution is

$$
\phi^{(0)}=\phi_{0} e^{-\left(h^{(0)}-z\right)}
$$

This leading-order solution corresponds to equilibrium compaction to which the porosity curve will tend when $t \rightarrow \infty$. The exponentially decreasing dependence of porosity on depth was found by Athy (1930) by fitting observed data of Paleozoic shales from Kansas and Oklahoma. Athy's porosity curve represents compaction equilibrium attained over a very long time span. Hedberg's (1936) porosity curve for the Tertiary shales in Venezuela is similar to Athy's curve.

Using (67), (61) becomes

$$
-\dot{h}^{(0)} \phi_{0} e^{-\left(h^{(0)}-z\right)}=\frac{\partial}{\partial z}\left\{\tilde{k}^{0}\left(1-\phi^{(0)}\right)^{2} \frac{1}{\phi^{(0)}}\left[\phi_{z}^{(1)}-\phi^{(1)}\right]\right\} .
$$

Integrating the above equation using (63), we have

$$
\phi_{z}^{(1)}-\phi^{(1)}-\frac{\dot{h}^{(0)} \phi_{0}\left(1-e^{z}\right) e^{-h^{(0)}} \phi^{(0)}}{\tilde{k}^{0}\left(1-\phi^{(0)}\right)^{2}}=0 .
$$

Using (69), (64), and (67), we obtain a relation for $\dot{h}^{(0)}$ :

$$
-\left(1-\dot{h}^{(0)}\right)\left(1-\phi_{0}\right)+\dot{h}^{(0)} \phi_{0}\left(1-e^{-h^{(0)}}\right)=0 .
$$

Integrating this equation, we have

$$
h^{(0)}=\left(1-\phi_{0}\right) t+\phi_{0}\left[1-e^{-h^{(0)}}\right] .
$$

Clearly, if $t$ is large, then $\exp \left[-h^{(0)}\right]<<1$; we thus have

$$
\dot{h}^{(0)} \approx 1-\phi_{0} \text {. }
$$

If $t$ is small, then $\exp \left[-h^{(0)}\right] \sim 1$, and

$$
\dot{h}^{(0)} \approx 1
$$


3.3. Strongly varying permeability: $\boldsymbol{m} \gg \mathbf{1}$. With $\tilde{k}^{0}=\left(\phi^{(0)} / \phi_{0}\right)^{m}$, we see that as $\phi^{(0)}$ decreases, $\tilde{k}^{0}$ can decrease dramatically if $m$ is relatively large. The perturbation expansions in (58) and (59) are valid only if $\lambda \tilde{k}>>1$, and we can therefore define a critical porosity $\phi^{*}$ when $\lambda \tilde{k}=1$. With $\tilde{k}=\left(\phi / \phi_{0}\right)^{m}$, we have

$$
\phi^{*}=\phi_{0} \exp \left[-\frac{1}{m} \ln \lambda\right]
$$

and with values $\phi_{0}=0.5, m=8, \lambda=100$, this critical value is $\phi^{*}=0.28$. As the slowly compacting layer thickens, we see that the perturbation solution is valid until $\phi^{(0)}=\phi^{*}$, which is when

$$
h^{(0)}=\Pi=\frac{1}{m} \ln \lambda \approx 0.58
$$

with the same values of $m$ and $\lambda$, and using (71), this occurs at $t=t^{*}$, where

$$
t^{*}=\frac{\Pi-\phi_{0}\left(1-e^{-\Pi}\right)}{1-\phi_{0}} \approx 0.71 .
$$

For $t>t^{*}$, the solution above can be expected to apply for $z>h^{(0)}-\Pi$.

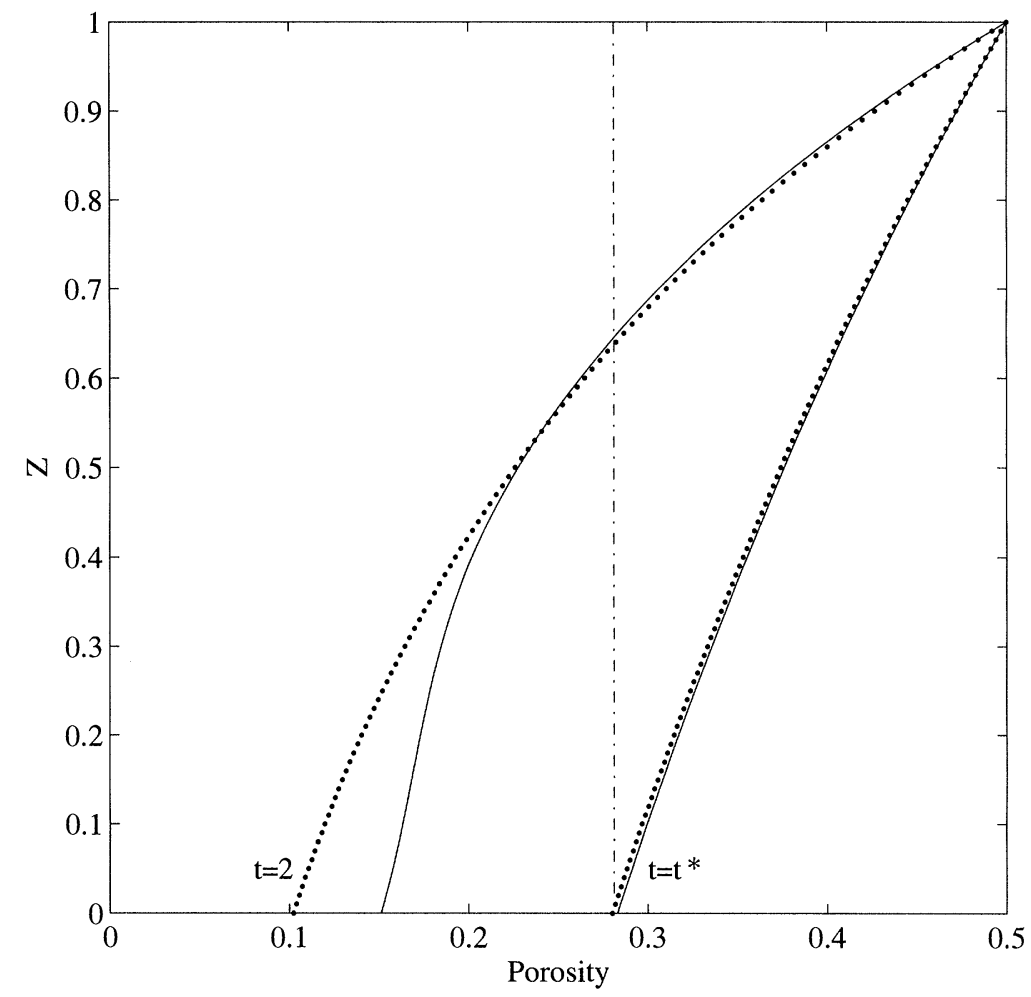

Fig. 4. Comparison of the large $\lambda$ solution, (67), with numerical results (solid) in terms of a normalized depth $Z=z / h$. For $t=t^{*}$ given by (76), the asymptotic result is accurate, but for $t>t^{*}$, it becomes invalid when $\phi<\phi^{*}=0.28$, for the values used here of $\lambda=100, \phi_{0}=0.5, m=8$.

A comparison of the solution with related numerical results is presented in Fig. 4. The comparison clearly shows that Athy's exponential porosity-depth relation (Athy 
(1930)) is valid only in the range of $0-0.58 d \mathrm{~km}$ in such sedimentary basins where the parameter $\lambda>>1$. If $d=1 \mathrm{~km}$, then the range is $0-580 \mathrm{~m}$. We now extend the analysis for $\lambda \gg 1$ to deal with this situation.

4. Thick layer sediments with $\phi<\phi^{*}$ and $t>t^{*}$. Note that, from its definition, $\phi^{*}<<1$ if $\lambda>>1$, so we must formally assume $m>>1$ in order to have $\phi^{*}$ of order 1 . Thus, we now consider a limit in which $m$ is large. We write the equations in terms of $\phi^{*}$ defined by (74) as follows:

$$
\phi_{t}=\frac{\partial}{\partial z}\left[\left(\frac{\phi}{\phi^{*}}\right)^{m}(1-\phi)^{2}\left(\frac{1}{\phi} \frac{\partial \phi}{\partial z}-1\right)\right]
$$

on $z=h$,

$$
\left(\frac{\phi}{\phi^{*}}\right)^{m}(1-\phi)\left(\frac{1}{\phi} \frac{\partial \phi}{\partial z}-1\right)=-(1-\dot{h})
$$

on $z=0$,

$$
\phi_{z}=\phi
$$

Now if $\phi>\phi^{*},\left(\phi / \phi^{*}\right)^{m}$ is exponentially large, and therefore, neglecting $\phi_{t}$ in $(77)$

$$
\left(\frac{\phi}{\phi^{*}}\right)^{m}(1-\phi)^{2}\left(\frac{1}{\phi} \frac{\partial \phi}{\partial z}-1\right) \approx-\left(1-\phi_{0}\right)(1-\dot{h}),
$$

using the boundary condition (78) at $z=h$. We still have (since $\left.\left(\phi / \phi^{*}\right)^{m} \gg 1\right)$

$$
\phi \approx \phi_{0} \exp [-(h-z)]
$$

from which $\phi_{t} \approx-\dot{h} \phi_{z}$, and an improved approximation to (79) is therefore, using this in (77),

$$
\left(\frac{\phi}{\phi^{*}}\right)^{m}(1-\phi)^{2}\left(\frac{1}{\phi} \frac{\partial \phi}{\partial z}-1\right) \approx \dot{h}\left(\phi_{0}-\phi\right)-\left(1-\phi_{0}\right)(1-\dot{h}) .
$$

This approximation, however, becomes invalid when $h-z \approx \Pi$, and specifically we define $\xi$ and $\Psi$ in the transition region near $h-z=\Pi$ by

$$
z=h-\Pi-\frac{\ln m}{m}+\frac{\xi}{m},
$$

$$
\phi=\phi^{*} \exp \left[\frac{1}{m}(-\ln m+\Psi)\right]
$$

from which it follows by a matching principle (Hinch, 1991) that $\Psi \sim \xi$ as $\xi \rightarrow \infty$. $\Psi$ satisfies the equation 


$$
\begin{aligned}
& \left(-\dot{h} \Psi_{\xi}+\frac{1}{m} \Psi_{t}\right) \phi_{\infty} \exp \left[\frac{1}{m}\left(\Psi-\Psi_{\infty}\right)\right] \\
= & \frac{\partial}{\partial \xi}\left[e^{\Psi}\left\{1-\phi_{\infty} \exp \left[\frac{1}{m}\left(\Psi-\Psi_{\infty}\right)\right]\right\}^{2}\left(\Psi_{\xi}-1\right)\right],
\end{aligned}
$$

where we define

$$
\phi_{\infty}=\phi^{*} \exp \left[\frac{1}{m}\left(-\ln m+\Psi_{\infty}\right)\right]
$$

and $\Psi_{\infty}$ will be defined below.

Neglecting terms of $O(1 / m)$ in $(83)$, we find

$$
K-\dot{h} \phi_{\infty} \Psi=e^{\Psi}\left(1-\phi_{\infty}\right)^{2}\left(\Psi_{\xi}-1\right)
$$

where

$$
K=\dot{h} \phi_{\infty} \Psi_{\infty}-\left(1-\phi_{\infty}\right)^{2} e^{\Psi_{\infty}}
$$

and $\Psi$ increases monotonically from $\Psi_{\infty}$ as $\xi \rightarrow-\infty$ to $O(\xi)$ as $\xi \rightarrow+\infty$. The value of $\Psi_{\infty}$ must now be found by matching to the solution below $z=h-\Pi$.

4.1. Prescription of $\dot{h}$. Before finding this solution, we can find $\dot{h}$ by comparing $(85)$ to $(81)$. We write the latter equation in terms of $\Psi$ and $\xi$, to obtain

$$
\begin{gathered}
\left\{1-\phi_{\infty} \exp \left[\frac{1}{m}\left(\Psi-\Psi_{\infty}\right)\right]\right\}^{2} e^{\Psi}\left(\Psi_{\xi}-1\right) \\
\approx m\left[\dot{h}\left\{\phi_{0}-\phi_{\infty} \exp \left[\frac{1}{m}\left(\Psi-\Psi_{\infty}\right)\right]\right\}-\left(1-\phi_{0}\right)(1-\dot{h})\right],
\end{gathered}
$$

whence

$$
\begin{gathered}
\left(1-\phi_{\infty}\right)^{2} e^{\Psi}\left(\Psi_{\xi}-1\right) \\
\approx m\left[\left(1-\phi_{\infty}\right) \dot{h}-\left(1-\phi_{0}\right)\right]-\dot{h} \phi_{\infty}\left(\Psi-\Psi_{\infty}\right)+\cdots,
\end{gathered}
$$

and in order that this matches to (85), we require (using the definition of $K$ in (86)) that

$$
\dot{h}=\frac{1-\phi_{0}}{1-\phi_{\infty}}-\frac{\left(1-\phi_{\infty}\right)^{2}}{m} e^{\Psi_{\infty}} \ldots
$$

Solution below the transition layer. We write the equation for $\Psi$, (83), in terms of $z$. It is

(90) $\Psi_{t} \phi_{\infty} \exp \left[\frac{1}{m}\left(\Psi-\Psi_{\infty}\right)\right]=\frac{\partial}{\partial z}\left[e^{\Psi}\left\{1-\phi_{\infty} \exp \left[\frac{1}{m}\left(\Psi-\Psi_{\infty}\right)\right]\right\}^{2}\left(\frac{1}{m} \Psi_{z}-1\right)\right]$ 


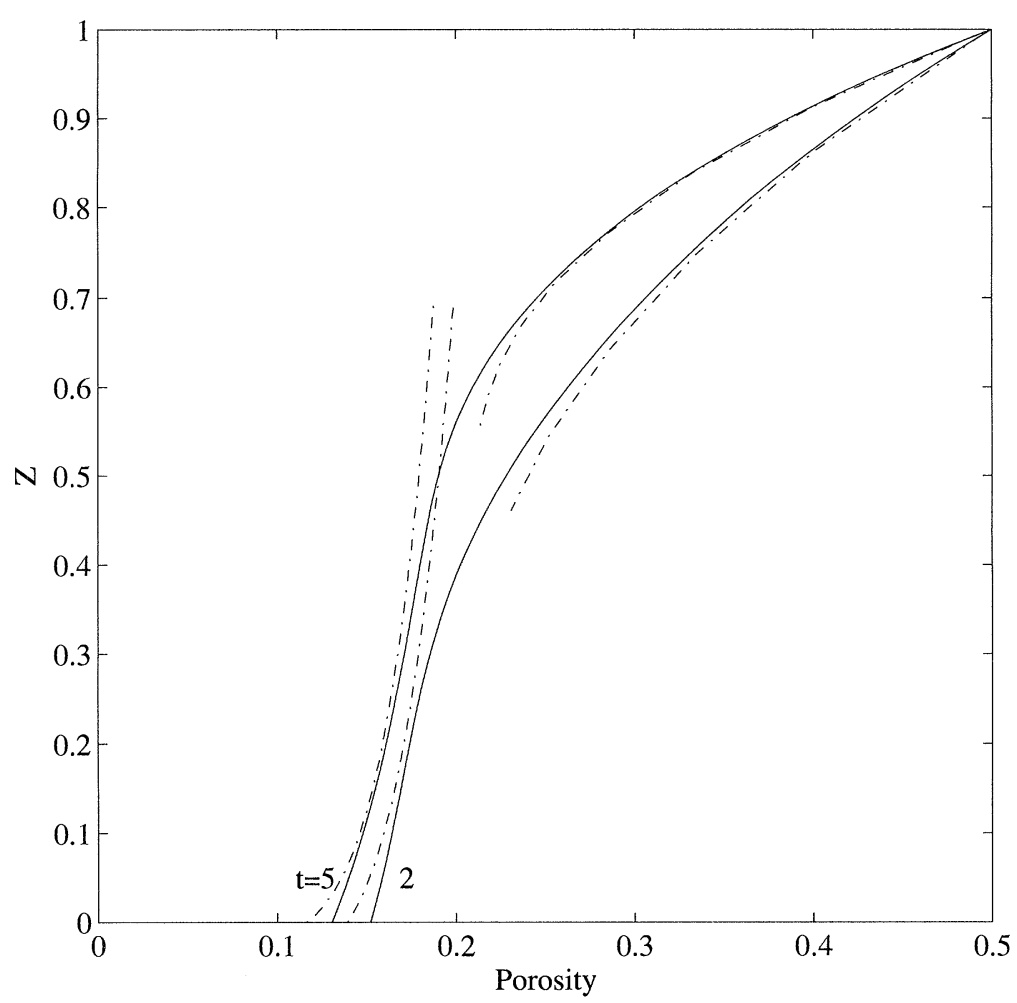

FIG. 5. Comparison of asymptotic solutions (81) and (94) (dashed lines) and numerical results (solid lines), for times $t=2$ and $t=5$, taking $\lambda=100$.

and at leading order,

$$
\phi_{\infty} \Psi_{t}+\left(1-\phi_{\infty}\right)^{2} e^{\Psi} \Psi_{z}=0
$$

a hyperbolic equation. The loss of the highest derivative means that only one boundary condition can be satisfied, and because the characteristics of (91) move upward, the appropriate condition to satisfy is that at $z=0$, which is $\Psi_{z}=m$. It seems that this condition is not correctly ordered, thus warranting consideration of a further basal boundary layer, but we show that by solving (91) together with $\Psi_{z}=m$ on $z=0$, we obtain a uniformly valid solution below $z=h-\Pi$.

We suppose that the initial data for (91) are

$$
\Psi=\Psi_{b}(\tau) \quad \text { when } z=0, t=\tau
$$

where, if $h=\Pi+\frac{1}{m} \ln m$ at $t=t_{0}\left(\approx t^{*}\right)$, then

$$
\Psi_{b}\left(t_{0}\right)=0,
$$

and we choose $\Psi_{b}(\tau)$ in order that $\Psi_{z}=m$ at $z=0$. The solution is easily found to be

$$
\Psi=\ln \left[\frac{1+m z}{1+m \frac{\left(1-\phi_{\infty}\right)^{2}}{\phi_{\infty}}\left(t-t_{0}\right)}\right]
$$

and $\Psi_{b}=0$. This satisfies the boundary condition on $z=0$; moreover, we see that $\left(\partial^{2} / \partial z^{2}\right) e^{\Psi}=0$ so that the diffusion term in (90) is of order $1 / m^{2}$, so long as $\Psi_{z}$ is 
of order 1. From (94), this is true for $z \sim O(1)$, so that (94) is (very) accurate away from the base. Near $z=0$, however, $\Psi_{z} \sim O(m)$, so the diffusion term is of order 1 there and not negligible. Since this is only true for $z \sim O(1 / m)$, the implication is that the solution (94) is uniformly accurate to $O(1 / m)$ for $\Psi$ in $z<h-\Pi$, with less accuracy near the base. This appears to be borne out in Figs. 5, 6, and 8 below.

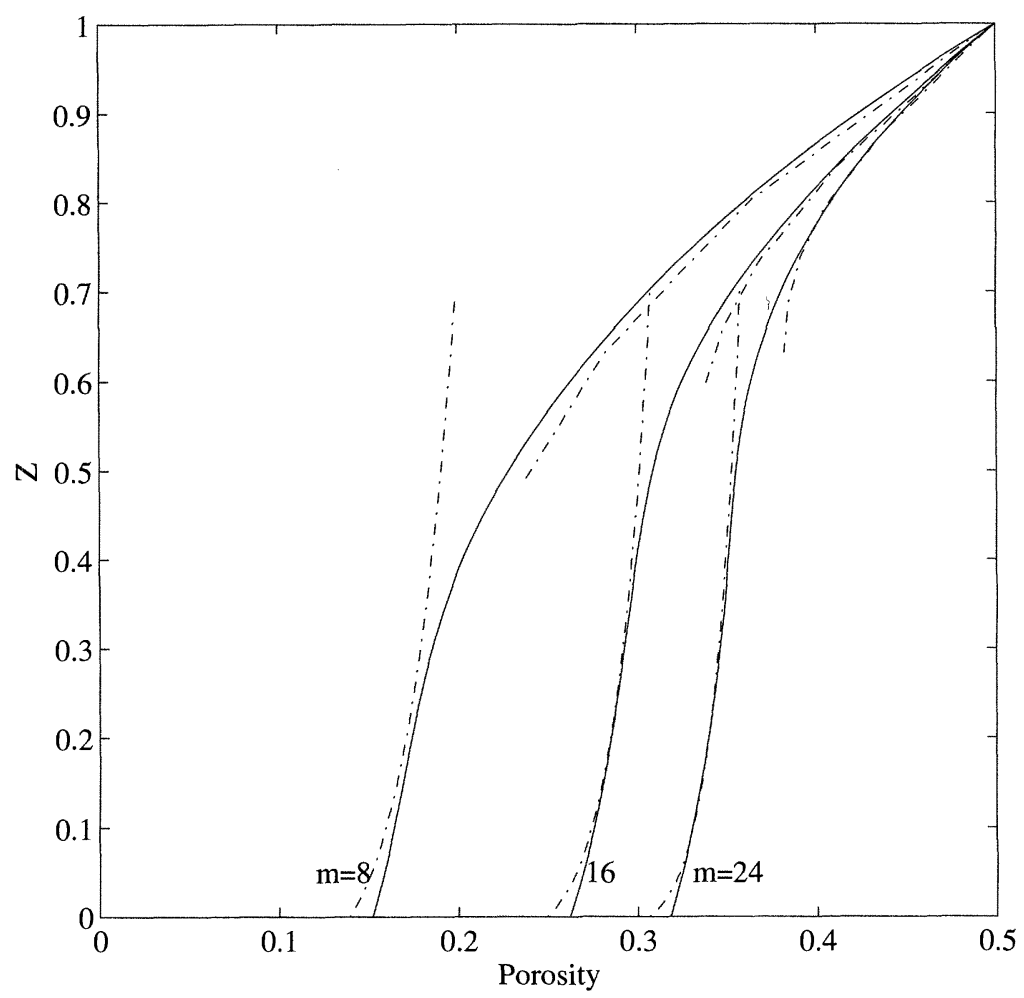

FIG. 6. Comparison of asymptotic solutions (81) and (94) (dashed lines) and numerical results (solid lines) with different values of $m: t=2$ in all plots, $\lambda=100$, and the curves correspond to values $m=8,16,24$.

We can now finally obtain $\Psi_{\infty}$ by matching (94) with (85) as $z \rightarrow h-\Pi$ and $\xi \rightarrow-\infty$. In fact, putting $z=h-\Pi-\frac{1}{m} \ln m+\frac{\xi}{m}$, (94) becomes

$$
\Psi=\ln \left[\frac{h-\Pi+\frac{1}{m}(1-\ln m+\xi)}{\frac{\left(1-\phi_{\infty}\right)^{2}}{\phi_{\infty}}\left(t-t_{0}\right)+\frac{1}{m}}\right],
$$

from which we require

$$
\Psi_{\infty}=\ln \left[\frac{\phi_{\infty}(h-\Pi)}{\left(1-\phi_{\infty}\right)^{2}\left(t-t_{0}\right)}\right]+o(1) .
$$

This completes the asymptotic solution.

Comparisons of the approximate solution derived above with the numerical solution are shown in Figs. 5 and 6 . For a value $\lambda=100$, Fig. 5 shows the comparison at times $t=2$ and $t=5$, while Fig. 6 compares approximate and exact solutions at $t=2$ for increasing values of $m$. The accuracy increases with $m$, as we expect. Figure 7 shows computed and approximate values of $h(t)$. 


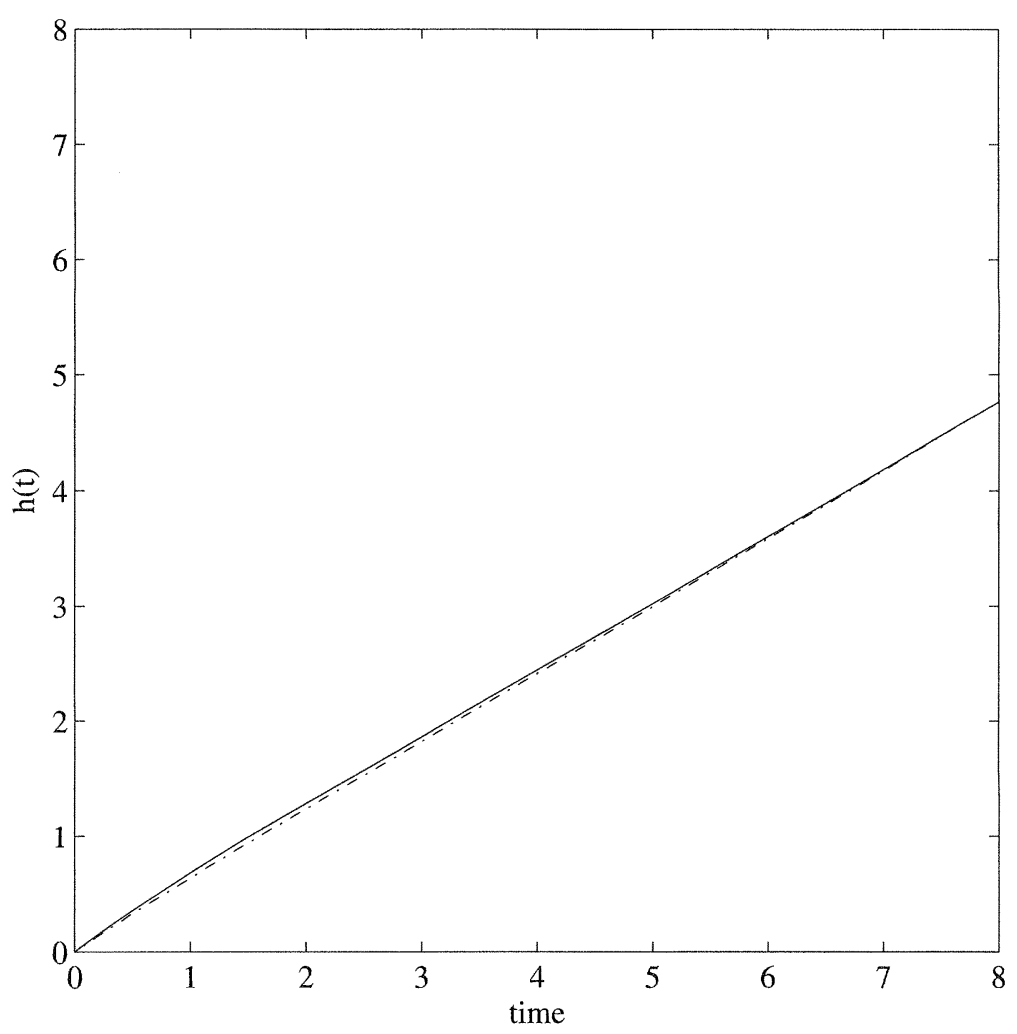

FIG. 7. Comparison of approximate $h(t)$ solution from (89) and (96) (dashed line) and numerical results (solid line); $\lambda=100$.

When $\lambda>>1$ and $\phi>\phi^{*}$, substituting (80) for $\phi$ into (56), we have

$$
\frac{\partial p_{\mathrm{ex}}}{\partial z}=0
$$

This equation with the boundary condition $p_{\mathrm{ex}}=0$ at the top $z=h(t)$ gives $p_{\mathrm{ex}}=0$ for the leading-order solution. This means excess pressure does not occur for short times or in the top region where $h-z<\Pi$. This region is clearly shown in Fig. 8 . For larger times, the approximate solution suggests that $\phi_{z}<<\phi$, whence

$$
\frac{\partial p_{\mathrm{ex}}}{\partial z} \approx-(1-\phi)
$$

which shows that the excess pore pressure develops at large times even if $\lambda>>1$.

The comparison of the numerical results for the pore pressure with that calculated from the asymptotic solutions (dashed lines) is shown in Fig. 8. The overpressure only develops in the lower region, while the pore pressure remains hydrostatic in the top region within a depth of order $\Pi$ from the surface.

5. Conclusions. In the absence of diagenesis and temperature effects, the generalized one-dimensional model of compaction given by Audet and Fowler (1992) reduces to a nonlinear diffusion equation in a domain with a moving boundary. When scaled, this model depends primarily on one dimensionless parameter $\lambda$, which is the ratio of the sedimentation time scale to the Darcy flow time scale. Thus, $\lambda>>1$ if 


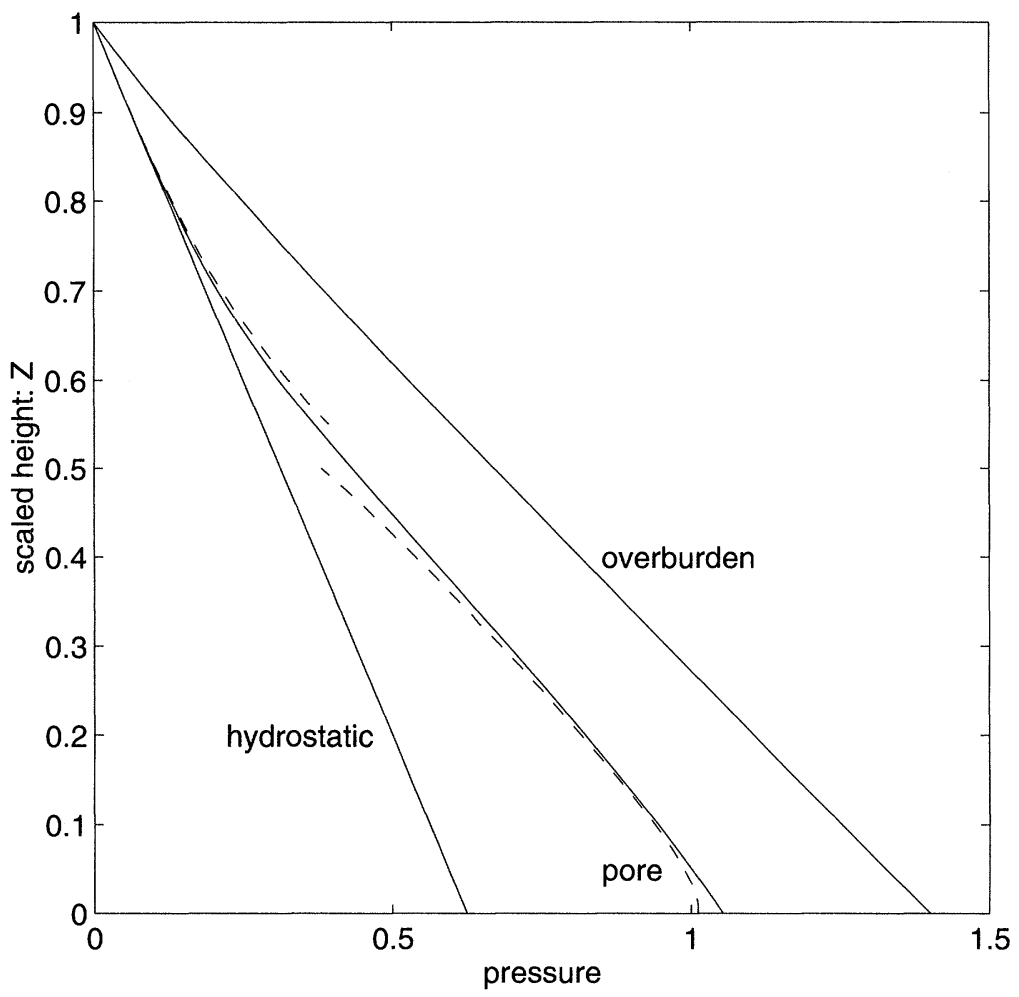

FiG. 8. Hydrostatic, pore, and overburden pressures at $t=5, \lambda=100$. Dashed lines are computed by using (81) and (94).

sedimentation is very slow, while $\lambda<<1$ if it is very fast. Realistically, both limits are possible, depending principally on the permeability. In addition, strong variability of the permeability through the exponent $m$ complicates the solution method.

In particular, we find that in the limit $\lambda<<1$ (slow compaction), the model can be simply analyzed by means of a boundary layer analysis at the sediment base. Essentially, sediment is added so fast that the porosity remains virgin except near the base, where compaction occurs. The pore pressure is then essentially lithostatic; that is, excess pore pressures exist over the whole domain.

The more interesting (and probably more relevant) case is when $\lambda>>1$ (fast compaction). For sufficiently small times (and thus also basin thicknesses), the porosity profile is exponential with depth, and the pore pressure has relaxed to a hydrostatic value. However, because of the large exponent $m$ in the permeability law $\tilde{k}=\left(\phi / \phi_{0}\right)^{m}$, we find that even if $\lambda>>1$, the product $\lambda \tilde{k}$ may become small at sufficiently large depths. In this case, there is a critical depth such that, when the basin thickness exceeds it, the porosity profile consists of an upper part near the surface where $\lambda \tilde{k}>>1$ and the exponential profile is attained, and a lower part where $\lambda \tilde{k}<<1$, and the porosity is higher than equilibrium. Straightforward asymptotic methods are difficult to implement because the limit $m>>1$ implies exponential asymptotics, but we use a hybrid method which appears to correspond accurately to numerical computations. 
The methods presented in this paper pave the path for the analysis of compaction in sedimentary basins when more complicated loading histories are studied, and also when more realistic phenomena are included, such as diagenesis, or state-dependent rheology (Schofield and Wroth (1968)).

\section{REFERENCES}

C. L. Angevine And D. L. Turcotte (1983), Porosity reduction by pressure solution-A theoretical model for quartz arenites, Geol. Soc. Amer. Bull., 94, pp. 1129-1124.

L. F. Aтнy (1930), Density, porosity, and compaction of sedimentary rocks, Amer. Ass. Petrol. Geol. Bull., 14, pp. 1-22.

D. M. Audet And A. C. Fowler (1992), A mathematical model for compaction in sedimentary basins, Geophys. J. Internat., 110, pp. 577-590.

J. BEAR AND Y. BACHMAT (1990), Introduction to Modelling of Transport Phenomena, Kluwer, Dordrecht, The Netherlands.

C. M BethKe AND F. CORBET (1988), Linear and nonlinear solutions for one-dimensional compaction flow in sedimentary basins, Water Resour. Res., 24, pp. 461-467.

R. S. Bishop (1979), Calculated compaction states of thick abnormally pressured shales, Amer. Petrol. Geol. Bull., 63, pp. 918-933.

J. D BREDEHOEFT AND B. B. HANSHAW (1968), On the maintenance of anomalous fluid pressures: I. Thick sedimentary sequences, Geol. Soc. Amer. Bull., 79, pp. 1097-1106.

H. S. Carslaw and J. C. Jaeger (1959), Conduction of Heat in Solids, 2nd ed., Oxford University Press, Oxford.

D. Eberl AND J. Hower (1976), Kinetics of illite formation, Geol. Soc. Amer. Bull., 87, pp. 13261330 .

A. C. Fowler (1985), A mathematical model of magma transport in the asthenosphere, Geophys. Astrophys. Fluid Dynam., 33, pp. 63-96.

A.C. Fowler (1990), A compaction model for melt transport in the earth's asthenosphere. Part I : The basic model, in Magma Transport and Storage, M.P. Ryan, ed., John Wiley, New York, pp. 3-14.

R. A. Freeze And J. A. Cherry (1979), Groundwater, Prentice-Hall, Englewood Cliffs, NJ.

R. E. GIBSON (1958), The progress of consolidation in a clay layer increasing in thickness with time, Géotechnique, 8, pp. 171-182.

R.E. Gibson, G.L. England, And M. J. L. Hussey (1967), The theory of one-dimensional consolidation of saturated clays, I. Finite non-linear consolidation of thin homogeneous layers, Canad. Geotech. J., 17, pp. 261-273.

R. E. Gibson, R. L. Schiffman, And K. W. CARGill (1981), The theory of one-dimensional consolidation of saturated clays, II. Finite non-linear consolidation of thick homogeneous layers, Canad. Geotech. J., 18, pp. 280-293.

H. D. Hedberg (1936), Gravitational compaction of clays and shales, Amer. J. Sci., 184, pp. 241287.

E. J. Hinch (1991), Perturbation Methods, Cambridge University Press, Cambridge, UK.

L. A. Keith And J. D. Rimstidt (1985), A numerical compaction model of overpressuring in shales, Math. Geol., 17, pp. 115-135.

I. Lerche (1990), Basin Analysis: Quantitative Methods, Vol. I, Academic Press, San Diego, CA.

X. LUO AND G. VASSEUR (1992), Contribution of compaction and aquathermal pressuring to geopressure and the influence of environmental conditions, AAPG Bull., 76, pp. 1550-1559.

D. P. MCKEnZIE (1984), The generation and compaction of partially molten rock, J. Petrol., 25, pp. $713-765$.

P. C. MEek AND J. Norbury (1982), Two-stage, two level finite difference schemes for non-linear parabolic equations, IMA J. Numer. Anal., 2, pp. 335-356.

H. H. Rieke AND C. V. Chilingarian (1974), Compaction of Argillaceous Sediments, Elsevier, Amsterdam.

A. N. Schofield And C. P. Wroth (1968), Critical State Soil Mechanics, McGraw-Hill, New York.

D. R. Scott And D. J. Stevenson (1984), Magma solitons, Geophys. Res. Lett., 11, pp. 1161-1164.

J. M. ShARP, JR., (1976), Momentum and energy balance equations for compacting sediments, Math. Geol., 8, pp. 305-322.

J. M. ShARP, JR. AND P. A. DOMEnico (1976), Energy transport in thick sequences of compacting sediment, Geol. Soc. Amer. Bull., 87, pp. 390-400.

Y. SHI AND C. Y. WANG (1986), Pore pressure generation in sedimentary basin, overloading versus 
aquathermal, J. Geophys. Res., 91, pp. 2153-2162.

A. W. Skempton (1960), Effective stress in soils, concrete and rocks, in Pore Pressure and Suction in Soils, Butterworth, London.

J. E. Sмiтн (1971), The dynamics of shale compaction and evolution in pore-fluid pressures, Math. Geol., 3, pp. 239-263.

K. Terzaghi (1943), Theoretical Soil Mechanics, John Wiley and Sons, New York.

M. WANGEN (1992), Pressure and temperature evolution in sedimentary basins, Geophys. J. Internat., 110 , pp. 601-613. 